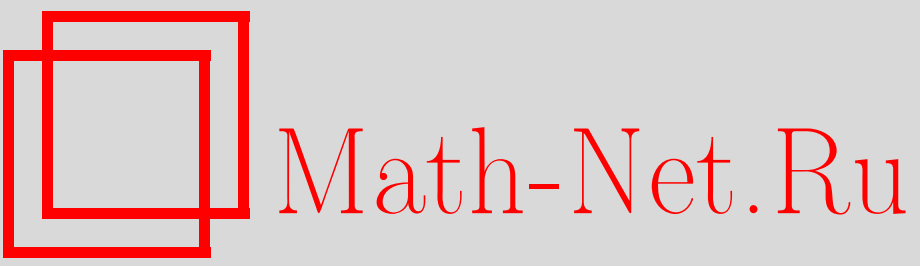

И. П. Богданов, В. А. Судаков, Н. Б. Топоров, Оптимизация загрузки упорядоченной совокупности летательных аппаратов, Матем. моделирование, 2020, том 32 , номер 4, 43-56

DOI: https://doi.org/10.20948/mm-2020-04-04

Использование Общероссийского математического портала Math-Net.Ru подразумевает, что вы прочитали и согласны с пользовательским соглашением

http: //www . mathnet.ru/rus/agreement

Параметры загрузки :

IP : 54.224 .60 .19

26 апреля 2023 г., 17:36:09 


\title{
ОПТИМИЗАЦИЯ ЗАГРУЗКИ УПОРЯДОЧЕННОЙ СОВОКУПНОСТИ ЛЕТАТЕЛЬНЫХ АППАРАТОВ
}

\author{
(C) 2020 2. И.П. Богданов ${ }^{1}$, В.А. Судаков ${ }^{1}$, Н.Б. Топоров ${ }^{2}$ \\ ${ }^{1}$ Институт прикладной математики им. М.В. Келдыша РАН \\ ${ }^{2}$ НИЦ «Институт имени Н.Е. Жуковского» \\ ilyabogdanov@mail.ru,sudakov@ws-dss.com,toporov@nrczh.ru
}

DOI: $10.20948 / \mathrm{mm}-2020-04-04$

Рассмотрена задача расчета оптимальной загрузки набора воздушных транспортных средств, упорядоченных в соответствии с заданной системой приоритетов. Для указанной задачи предложена формализация в виде задачи смешанного целочисленного линейного программирования, требующая вдвое меньше бинарных переменных для записи условий отсутствия пересечения грузов по сравнению с подходами, представленными в литературе. Разработана технология точного решения указанной задачи, реализованная в форме открытого веб-сервиса. Описаны основные свойства и практическая значимость предложенной технологии.

Ключевые слова: загрузка воздушных транспортных средств, упаковка контейнеров, смешанное целочисленное линейное программирование.

\section{LOADING OPTIMIZATION OF AN ORDERED SET OF AIRCRAFTS}

\author{
I.P. Bogdanov ${ }^{1}$, V.A. Sudakov ${ }^{1}$, N.B. Toporov ${ }^{2}$ \\ ${ }^{1}$ Keldysh Institute of Applied Mathematics of RAS \\ 2 The National Research Center «Zhukovsky Institute»
}

This paper deals with the problem of optimal loading of the air vehicles set, ordered in accordance with the given priority rules. The regarded problem is formalized as a mixedinteger linear programming task. Proposed formalization requires twice less binary variables to formalize the conditions for the absence of the intersections of loaded objects in comparison with approaches, regarded in literature. The exact solution technology, designed in the form of a public web-service, is presented. Features and practical significance of the developed technology are described.

Key words: aircraft loading, container packing, mixed-integer linear programming. 


\section{1. Введение}

Государственные и коммерческие организации, деятельность которых подразумевает необходимость планирования и осуществления доставки значительных объемов грузов, ежедневно сталкиваются с необходимостью выбора наилучшего способа расположения большого количества различных предметов внутри трехмерных контейнеров (складов, вагонов, фур, трюмов кораблей и др.). «Наилучший способ расположения» может соответствовать варианту укладки, при котором:

- все имеющиеся грузы размещают в минимально возможном количестве контейнеров;

- в имеющиеся контейнеры загружают совокупность предметов, характеризующуюся максимальным суммарным объемом (суммарной массой, количеством, полезностью);

- средство транспортировки, в котором осуществляется доставка, обладает наилучшими инерционными характеристиками и т.д.

При этом на практике необходимо учитывать условия, связанные с ограничениями на вместимость и грузоподъемность контейнеров, устойчивостью и сбалансированностью размещаемых объектов, группировкой и взаимным расположением предметов относительно друг друга (а также относительно стенок и пола контейнеров), очередностью погрузки и разгрузки и т.д. [1].

Неоптимальное расположение объектов может привести к существенным дополнительным финансовым затратам (на аренду помещений, фрахт судов, горюче-смазочные материалы и др.), увеличению продолжительности и сложности погрузочно-разгрузочных работ, а также повышению рисков повреждения предметов при транспортировке. Таким образом, возникает класс задач условной оптимизации, называемых в литературе задачами оптимальной упаковки, загрузки либо укладки контейнеров. В настоящем исследовании рассмотрена задача размещения грузов на бортах заданной совокупности летательных аппаратов (далее - ЛА), упорядоченных в соответствии с возрастанием приоритета. Требуется максимизировать число объектов на бортах наиболее приоритетных ЛА, а также суммарную массу всех загруженных предметов (так как в общем случае некоторые предметы могут остаться неупакованными). При этом грузы не должны выходить за пределы ЛА, а также пересекаться друг с другом. Учитывая, что транспортировка осуществляется воздушным путем, дополнительно должны выполняться ограничения на расположение центров масс наборов размещаемых объектов.

В статье приводится аналитическое описание пространства рассмотренной задачи (будем называть ее задачей оптимизации загрузки воздушно- 
го транспорта или ОЗВТ), позволяющее формализовать введенные выше условия в виде линейных уравнений и неравенств, а саму задачу - в виде задачи смешанного целочисленного линейного программирования (далее СЦЛП). Предложена технология поиска ее точного решения, реализованная в форме открытого сервиса, доступного в сети Интернет. Разработанная технология позволяет вычислять точное решение задачи ОЗВТ для случая небольшого числа контейнеров и грузов, и, таким образом, может быть применена для проверки качества решения, получаемого с помощью эвристических методов. Для случая большей размерности точное решение может быть оценено методами, использующими построенное аналитическое описание задачи.

В перспективе полученные результаты могут стать основой для разработки отдельных функциональных модулей систем поддержки принятия решений в сфере организации авиационных грузовых перевозок. Внедрение указанных систем позволит повысить эффективность использования авиационной техники и инфраструктуры за счет рационального выбора ЛА и оптимизации размещения грузов на борту выбранных ЛА. Кроме того, уменьшение числа полетов окажет положительное влияние на окружающую среду вследствие сокращения эмиссии вредных веществ и снижения уровня шума.

\section{2. Обзор основных направлений исследований}

В литературе рассматривается существенное количество типов задач упаковки (загрузки, укладки) контейнеров, различающихся [2]:

- оптимизируемыми целевыми функциями;

- количеством контейнеров: укладка может осуществляться в единственный контейнер либо в совокупность нескольких контейнеров;

- характеристиками совокупности контейнеров: множество контейнеров может либо состоять из контейнеров одинаковых формы и размеров, либо быть слабо неоднородным (т.е. состоять из контейнеров, относящихся к одному из немногочисленных типов, при том, что количество контейнеров каждого типа достаточно велико), либо быть сильно неоднородным (в случае, если среди контейнеров мало или нет одинаковых);

- характеристиками совокупности грузов: множество грузов может либо состоять из предметов одинаковых формы и размеров, либо быть слабо неоднородным, либо быть сильно неоднородным;

- формой контейнеров и/или грузов: как правило, считается, что контейнеры и грузы имеют форму прямоугольных параллелепипедов (кубоидов), примеры исключений можно найти в работе [3], посвященной упаков- 
ке предметов мебели, и в статье [4], где предполагается, что контейнеры могут иметь форму усеченных кубоидов;

- набором учитываемых практических ограничений [1] и т.д.

Классификация различных постановок задач упаковки контейнеров, а также обширный обзор методов их решения (163 научно-технических источника) приведены в [2].

Задачи оптимальной загрузки относятся к классу NP-трудных задач [5], и для нахождения их точного решения в общем случае требуется полный перебор возможных вариантов распределения предметов по контейнерам. В связи с этим в настоящее время активно развиваются эвристические подходы, позволяющие относительно быстро строить приближенные решения. Пример эвристического метода упаковки единственного контейнера, основанного на алгоритмах комбинирования предметов в блоки-кубоиды и сведения задачи трехмерной укладки к задаче двумерной укладки, приведен в [6]. В случае загрузки нескольких контейнеров широко применяют следующие стратегии размещения грузов [7]:

- упаковка контейнеров осуществляется последовательно (упаковка очередного контейнера начинается после заполнения предыдущего) - например, см. [8], где данная стратегия используется совместно с механизмами динамической приоритизации грузов;

- осуществляется предварительное распределение наборов грузов по контейнерам (например, в [9] для этой цели применяется поиск с запретами), после чего применяются алгоритмы укладки предметов в каждый отдельный контейнер и алгоритмы перераспределения грузов между контейнерами;

- одновременно осуществляется упаковка нескольких контейнеров.

В $[7,10]$ задача упаковки нескольких контейнеров сформулирована как задача целочисленного линейного программирования, при этом для каждого рассматриваемого типа контейнера полагаются известными так называемые «паттерны» - способы размещения в них грузов имеющихся типов. Так как при значительном количестве типов контейнеров и грузов число всех возможных способов размещения будет очень велико, применяются эвристические алгоритмы построения некоторого достаточно представительного набора «паттернов» (таким образом, данный подход не гарантирует нахождения точного решения).

Наряду с эвристическими подходами, разрабатываются методы, позволяющие строить точное решение для случая небольшого количества контейнеров и грузов. Как правило, предлагается аналитическая модель разме- 
щения предметов, позволяющая записать задачу загрузки в форме задачи СЦЛП, точное решение которой может быть найдено, например, методом ветвей и границ. В [11-13] рассматривается модель размещения, в которой переменные, определяющие расположение объекта (данные переменные соответствуют координатам одного из углов либо центра масс объекта), являются непрерывными. Для формализации условий отсутствия пересечения грузов для каждой пары предметов вводятся переменные-индикаторы того, что один из грузов находится левее/правее, выше/ниже, сзади/спереди другого груза. В [4] используются аналогичные переменные-индикаторы отсутствия пересечений, но переменные, определяющие расположение объекта, полагаются целочисленными. В [14] представлен подход, основанный на дискретизации пространства (т.е. введении конечной сетки) внутри контейнера. Размещение предметов, определяемое размещением их левых нижних передних углов, возможно только в узлах введенной сетки. В целях формализации условий отсутствия пересечения объектов для каждого типа груза и каждой упорядоченной пары точек конечной сетки априори вычисляется отображение-индикатор того, что область пространства, занимаемая размещенным в первой точке грузом указанного типа (за исключением его правой, верхней и задней граней), содержит вторую точку.

\section{3. Постановка задачи}

Рассмотрим следующий вариант задачи упаковки сильно неоднородной совокупности предметов в сильно неоднородное множество контейнеров. Имеется набор грузов, предназначенных для отправки с помощью самолетов либо вертолетов, а также совокупность контейнеров (соответствующих грузовым отсекам ЛА), в которых предполагается осуществлять доставку указанных грузов (в настоящем исследовании будем считать, что в каждом ЛА есть единственный грузовой отсек). Предполагается, что контейнеры и грузы имеют форму прямоугольных параллелепипедов (произвольных размеров), при этом загрузка отдельных предметов в ЛА осуществляется таким образом, чтобы боковые грани кубоидов-грузов были параллельны/перпендикулярны стенкам контейнеров. Для простоты будем считать, что грузы нельзя поворачивать. Предметы, помещенные в контейнеры, не должны выходить за границы контейнеров, а также не должны пересекаться друг с другом. Для каждого груза задана его масса и габариты (длина, ширина и высота). Для каждого контейнера заданы его грузоподъемность, габариты, а также диапазон, в котором должен находиться центр масс совокупности всех грузов, размещенных в данном контейнере (для простоты в дальнейшем будем предполагать, что центры масс отдельных 
предметов совпадают с их геометрическими центрами). Кроме того, предполагается, что контейнерам присвоены приоритеты, например, рассчитанные с помощью комбинированного метода [15]. Содержательно задача ОЗВТ состоит в том, чтобы, с одной стороны, разместить как можно больше предметов в более приоритетных контейнерах, а с другой стороны, в том, чтобы упаковать в контейнеры грузы, характеризующиеся наибольшей суммарной массой.

Построим модель размещения грузов в контейнерах, которая позволит формализовать рассматриваемую задачу как задачу СЦЛП. Так как для случая сильно неоднородных множеств контейнеров и грузов дискретизация пространства (аналогично [14]) может привести к задаче неприемлемо большой размерности, то целесообразно строить формализацию, схожую с предложенными в [11-13]. Введем прямоугольную систему координат в трехмерном пространстве. По оси $x$ будем откладывать длину, по оси $y$ - ширину, а по оси $z$ - высоту контейнеров и грузов. Добавим в имеющееся множество контейнеров дополнительный воображаемый контейнер с наименьшим приоритетом. Длина указанного контейнера (в дальнейшем будем его назвать виртуальным, а остальные контейнеры - реальными) равна сумме длин всех грузов, ширина равна ширине самого широкого груза, а высота равна высоте самого высокого груза. Грузоподъемность виртуального контейнера равна сумме масс всех грузов. Центр масс совокупности предметов, загруженных в данный контейнер, может находиться в любой его точке. В виртуальном контейнере окажутся все грузы, которые не получится разместить в реальных контейнерах. Расположим все контейнеры один за другим слева направо вдоль оси $x$ в порядке увеличения приоритета таким образом, чтобы грани контейнеров были параллельны координатным плоскостям, а левый нижний задний угол каждого контейнера располагался на оси $x$. При данном размещении самое правое положение будет занимать самый приоритетный реальный контейнер, а самое левое - виртуальный контейнер, левый нижний задний угол которого будет находиться в начале координат. Графическая схема расположения контейнеров приведена на рис.1.

Пронумеруем все контейнеры в соответствии с возрастанием приоритета (номер 1 получит виртуальный контейнер). Введем следующие обозначения: $g$ - количество контейнеров, $n-$ количество грузов, $m_{i}$ - масса $i$-го груза, $M_{j}$ - грузоподъемность $j$-го контейнера, $l_{i}, w_{i}, h_{i}$ - длина, ширина и высота $i$-го груза, $L_{j}, W_{j}, H_{j}$ - длина, ширина и высота $j$-го контейнера, $X_{j}^{\min }, X_{j}^{\max }, Y_{j}^{\min }, Y_{j}^{\max }, Z_{j}^{\min }, Z_{j}^{\max }$ - границы диапазона допустимого по- 
ложения центра масс грузов, размещенных в $j$-м контейнере (рассчитанные относительно левого нижнего заднего угла контейнера), $x_{i}, y_{i}, z_{i}$ - координаты размещения левого нижнего заднего угла $i$-го груза, $r_{i j}-$ индикатор размещения $i$-го груза в $j$-м контейнере, $K$ - константа, заведомо большая любых координат, $L_{j}^{*}$ - координата левой грани $j$-го контейнера по оси $x$.

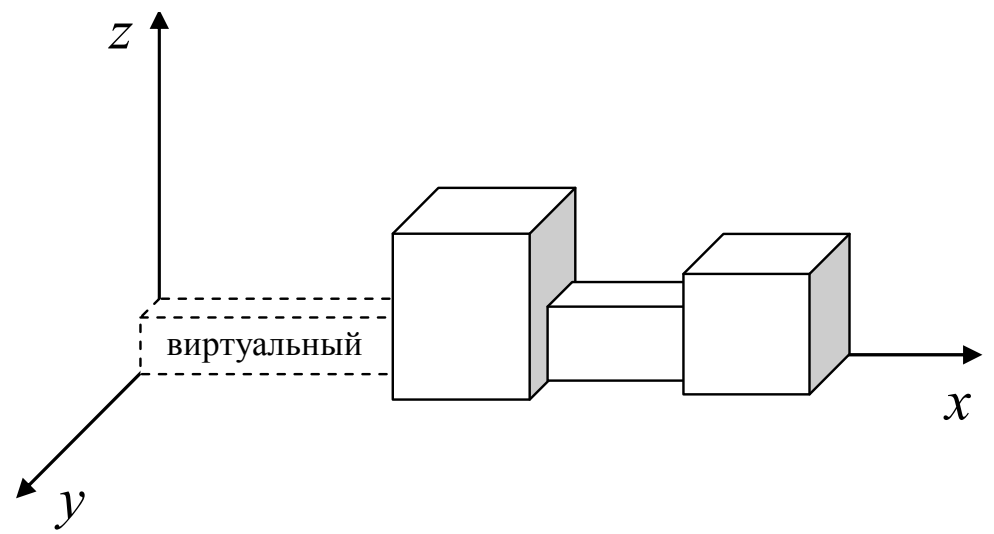

Рис.1. Схема расположения контейнеров.

Формализуем ограничения задачи в виде системы линейных уравнений и неравенств.

Каждый груз должен быть размещен в одном и только одном контейнере:

$$
\forall i=1, \ldots, n: \sum_{j=1}^{g} r_{i j}=1 .
$$

Ограничения на грузоподъемность и емкость контейнеров имеют вид

$$
\forall j=1, \ldots, g: \sum_{i=1}^{n} m_{i} r_{i j} \leq M_{j}, \quad \sum_{i=1}^{n}\left(l_{i} \cdot w_{i} \cdot h_{i}\right) r_{i j} \leq L_{j} \cdot W_{j} \cdot H_{j} .
$$

Грузы не должны выходить за границы контейнеров, т.е. для любых $i=1, \ldots, n, j=1, \ldots, g$

$$
\begin{aligned}
& x_{i}+K\left(1-r_{i j}\right) \geq L_{j}^{*}, \quad x_{i}+l_{i}-K\left(1-r_{i j}\right) \leq L_{j}^{*}+L_{j}, \\
& y_{i} \geq 0, y_{i}+w_{i}-K\left(1-r_{i j}\right) \leq W_{j}, \quad z_{i} \geq 0, \quad z_{i}+h_{i}-K\left(1-r_{i j}\right) \leq H_{j} .
\end{aligned}
$$

Условия на границы допустимого положения центров масс совокупностей загруженных предметов для каждого контейнера $j=1, \ldots, g$ можно записать в виде

$$
\left(X_{j}^{\min }+L_{j}^{*}\right) \sum_{v=1}^{n} m_{v} r_{v j} \leq \sum_{i=1}^{n}\left(m_{i}\left(p_{i j}^{x}+r_{i j} l_{i} / 2\right)\right) \leq\left(X_{j}^{\max }+L_{j}^{*}\right) \sum_{v=1}^{n} m_{v} r_{v j},
$$




$$
\begin{aligned}
& Y_{j}^{\min } \sum_{v=1}^{n} m_{v} r_{v j} \leq \sum_{i=1}^{n}\left(m_{i}\left(p_{i j}^{y}+r_{i j} w_{i} / 2\right)\right) \leq Y_{j}^{\max } \sum_{v=1}^{n} m_{v} r_{v j}, \\
& Z_{j}^{\min } \sum_{v=1}^{n} m_{v} r_{v j} \leq \sum_{i=1}^{n}\left(m_{i}\left(p_{i j}^{z}+r_{i j} h_{i} / 2\right)\right) \leq Z_{j}^{\max } \sum_{v=1}^{n} m_{v} r_{v j},
\end{aligned}
$$

где $p_{i j}^{x}, p_{i j}^{y}, p_{i j}^{z}$ - вспомогательные непрерывные переменные, на которые мы заменяем произведения $r_{i j} x_{i}, r_{i j} y_{i}, r_{i j} z_{i}$ путем добавления следующих неравенств:

$$
\begin{array}{lll}
p_{i j}^{x} \leq K r_{i j}, & p_{i j}^{x} \leq x_{i}, \quad p_{i j}^{x} \geq x_{i}-K\left(1-r_{i j}\right), & p_{i j}^{x} \geq 0, \\
p_{i j}^{y} \leq K r_{i j}, & p_{i j}^{y} \leq y_{i}, \quad p_{i j}^{y} \geq y_{i}-K\left(1-r_{i j}\right), & p_{i j}^{y} \geq 0, \\
p_{i j}^{z} \leq K r_{i j}, & p_{i j}^{z} \leq z_{i}, \quad p_{i j}^{z} \geq z_{i}-K\left(1-r_{i j}\right), \quad p_{i j}^{z} \geq 0 .
\end{array}
$$

Условия на отсутствие пересечений грузов могут быть записаны с помощью следующей системы неравенств $\left(\forall i_{1}=1, \ldots, n-1, i_{2}=i_{1}+1, \ldots, n\right)$ :

$$
\begin{aligned}
& x_{i_{1}}-x_{i_{2}}-K a_{i_{1}, i_{2}}-K b_{i_{1}, i_{2}}-K c_{i_{1}, i_{2}} \leq-l_{i_{1}}, \\
& x_{i_{2}}-x_{i_{1}}-K\left(1-a_{i_{1}, i_{2}}\right)-K b_{i_{1}, i_{2}}-K c_{i_{1}, i_{2}} \leq-l_{i_{2}}, \\
& y_{i_{1}}-y_{i_{2}}-K a_{i_{1}, i_{2}}-K\left(1-b_{i_{1}, i_{2}}\right)-K c_{i_{1}, i_{2}} \leq-w_{i_{1}}, \\
& y_{i_{2}}-y_{i_{1}}-K\left(1-a_{i_{1}, i_{2}}\right)-K\left(1-b_{i_{1}, i_{2}}\right)-K c_{i_{1}, i_{2}} \leq-w_{i_{2}}, \\
& z_{i_{1}}-z_{i_{2}}-K a_{i_{1}, i_{2}}-K b_{i_{1}, i_{2}}-K\left(1-c_{i_{1}, i_{2}}\right) \leq-h_{i_{1}}, \\
& z_{i_{2}}-z_{i_{1}}-K\left(1-a_{i_{1}, i_{2}}\right)-K b_{i_{1}, i_{2}}-K\left(1-c_{i_{1}, i_{2}}\right) \leq-h_{i_{2}}, \\
& a_{i_{1}, i_{2}}+2 b_{i_{1}, i_{2}}+4 c_{i_{1}, i_{2}} \leq 5,
\end{aligned}
$$

где $a_{i_{1}, i_{2}}, b_{i_{1}, i_{2}}, c_{i_{1}, i_{2}}$ - вспомогательные бинарные переменные.

Качество варианта загрузки определяется координатами расположения грузов по оси $x$ (чем правее расположен груз, тем лучше), а также суммарной массой грузов, размещенных в реальных контейнерах. Тогда целевую функцию задачи ОЗВТ можно записать в виде

$$
\alpha\left(\sum_{i=1}^{n} x_{i}\right)+\beta\left(\sum_{i=1}^{n} \sum_{j=2}^{g} m_{i} r_{i j}\right),
$$

где $\alpha$ и $\beta$-весовые коэффициенты. 
Таким образом, задача ОЗВТ представляет собой задачу поиска максимума функции (18) по непрерывным переменным $x_{i}, y_{i}, z_{i}, p_{i j}^{x}, p_{i j}^{y}, p_{i j}^{z}$ $(i=1, \ldots, n, j=1, \ldots, g)$ и бинарным переменным $r_{i j}, a_{i_{1}, i_{2}}, b_{i_{1}, i_{2}}, c_{i_{1}, i_{2}}(i=1, \ldots, n$, $\left.j=1, \ldots, g, i_{1}=1, \ldots, n-1, i_{2}=i_{1}+1, \ldots, n\right)$ при ограничениях (1)-(17).

\section{4. Предлагаемая технология решения задачи ОЗВТ}

Построенная формализация задачи ОЗВТ относится к классу задач СЦЛП, для которых существуют алгоритмы, гарантирующие нахождение точного решения (хотя поиск оптимума может потребовать значительного времени и ресурсов). На практике наиболее широко используются алгоритмы ветвей и отсечений, основанные на комбинированном применении метода ветвей и границ [16] и метода отсекающих плоскостей [17]. Указанные методологии реализованы в представленных в настоящей момент на рынке коммерческих системах (например, IBM ILOG CPLEX, Gurobi, FICO Xpress и др.), а также в ряде некоммерческих комплексов (например, SCIP, MINTO, GLPK, COIN-OR branch and cut и др. [18]).

В рамках настоящего исследования была сформирована технология решения задачи ОЗВТ, основанная на использовании системы веб-сервисов поддержки принятия решений WS-DSS (http://ws-dss.com) и оптимизатора SCIP (https://scip.zib.de/), применяющего для нахождения оптимума алгоритм ветвей и отсечений, метод генерации столбцов, а также ряд эвристических способов поиска допустимых решений, позволяющих сократить пространство перебора [19]. В целях верификации разработанного программного обеспечения были проведены тестовые испытания расчетной системы на примерах, содержащих до 10 грузов и до 5 контейнеров (включая виртуальный). Сопоставление результатов численного моделирования с осуществленным вручную перебором продемонстрировало общую адекватность результатов работы предложенной технологии.

Ниже представлены результаты численного моделирования для задачи размещения 6 грузов в 4 реальных контейнерах. Контейнеры упорядочены и пронумерованы в соответствии с возрастанием приоритета (виртуальный контейнер получает номер 1, а наиболее приоритетный реальный контейнер - номер 5). Значения параметров реальных контейнеров - грузоподъемности (в килограммах) и габаритов (в метрах) - приведены в табл.1. Параметры виртуального контейнера автоматически рассчитываются в соответствии с правилами, описанными в разделе 3. Значения параметров грузов - массы (в килограммах) и габаритов (в метрах) - приведены в табл.2. Весовые коэффициенты целевой функции полагаются равными 0.5 : $\alpha=\beta=0.5$. 
Таблица 1. Параметры контейнеров.

\begin{tabular}{|c|c|c|c|c|}
\hline Номер контейнера & Грузоподъемность & Длина & Ширина & Высота \\
\hline 2 & 1200 & 7 & 7 & 7 \\
\hline 3 & 1200 & 3 & 3 & 3 \\
\hline 4 & 64.1 & 5 & 5 & 5 \\
\hline 5 & 1200 & 10 & 10 & 10 \\
\hline
\end{tabular}

Таблица 2. Параметры грузов.

\begin{tabular}{|c|c|c|c|c|}
\hline Номер груза & Масса & Длина & Ширина & Высота \\
\hline 1 & 500 & 10 & 10 & 5 \\
\hline 2 & 250 & 10 & 5 & 5 \\
\hline 3 & 125 & 5 & 5 & 5 \\
\hline 4 & 125 & 5 & 5 & 5 \\
\hline 5 & 64 & 4 & 4 & 4 \\
\hline 6 & 1 & 1 & 1 & 1 \\
\hline
\end{tabular}

контейнер 5

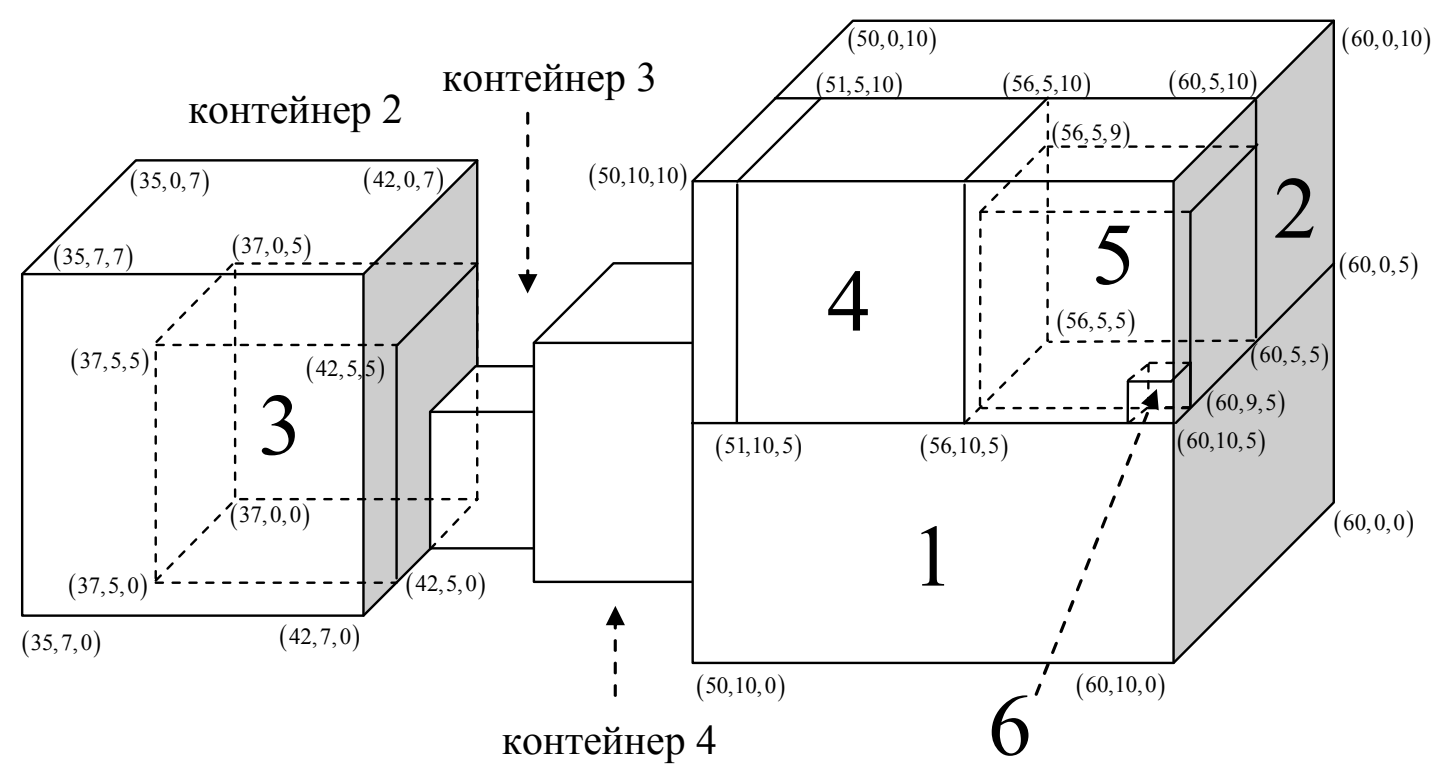

Рис.2. Распределение грузов (без учета ограничений на положение центров масс).

На рис.2 схематично показано распределение предметов по контейнерам в случае, когда границы диапазонов допустимого положения центров масс наборов грузов совпадают с границами контейнеров, а на рис. 3 - распределение предметов по контейнерам при дополнительном требовании размещения центров масс совокупностей упакованных грузов в геометрических центрах реальных контейнеров.

Численное моделирование проводилось на сервере AWS t2.small (1 vCPU, 2 Gb RAM, 20 Gb SSD). Результаты экспериментов продемонстрировали, что время поиска решения существенно зависит не только от количе- 
ства контейнеров и грузов, но и от соотношения их габаритов. В качестве иллюстрации в табл.3 приведено время расчета оптимального распределения 10 одинаковых грузов размера $1 \times 1 \times 1$ (здесь и далее размеры указаны в метрах) по 4 реальным контейнерам при различных значениях длины наиболее вместительного реального контейнера.

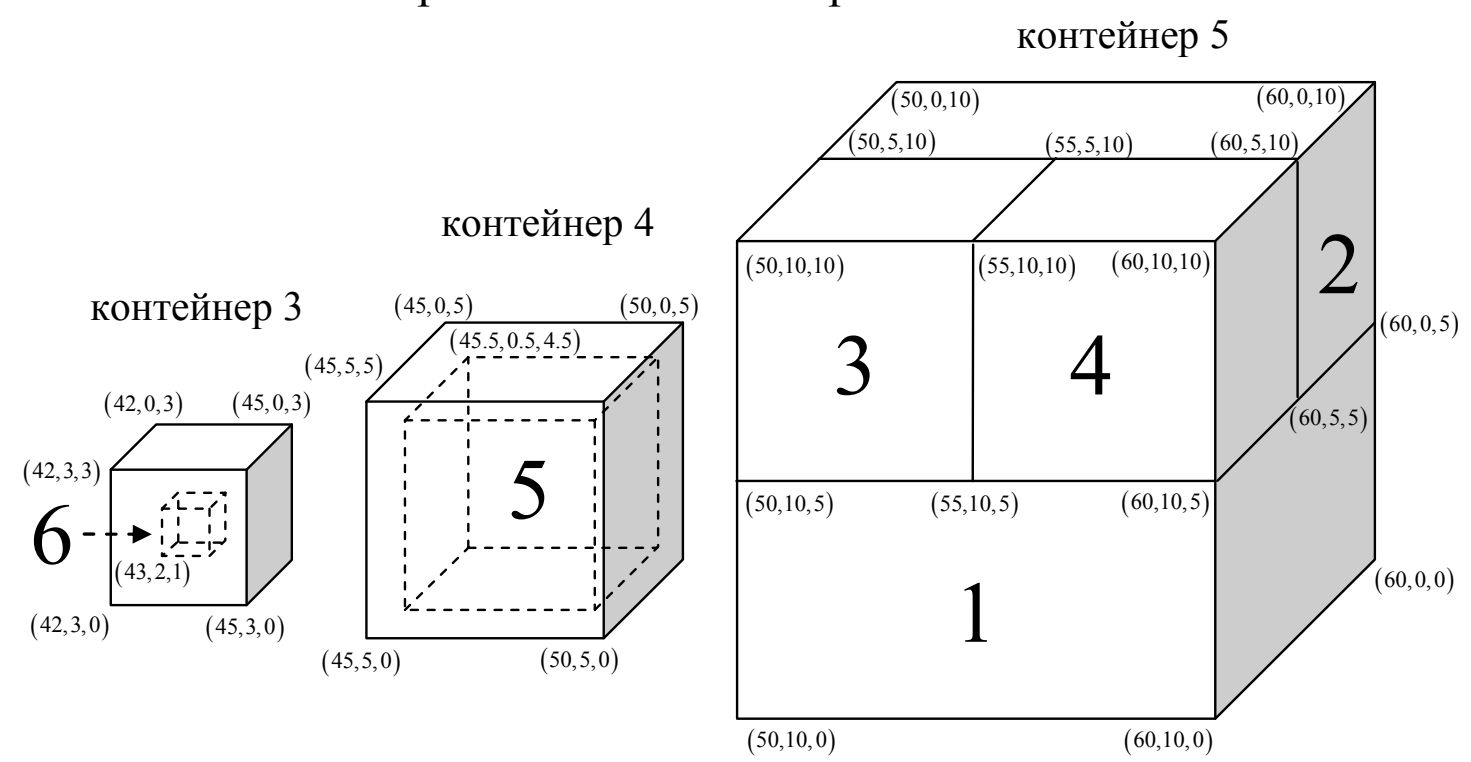

Рис.3. Распределение грузов (с учетом ограничений на положение центров масс).

Таблица 3. Время расчета для тестовых примеров.

\begin{tabular}{|c|c|c|}
\hline Габариты грузов & Габариты реальных контейнеров & Время расчета \\
\hline \multirow{2}{*}{$1 \times 1 \times 1(10$ штук $)$} & $\begin{array}{c}10.2 \times 1.2 \times 1.2,2.2 \times 1.2 \times 1.2 \\
2.2 \times 1.2 \times 1.2,1.2 \times 1.2 \times 1.2\end{array}$ & $<0.5$ секунды \\
\hline \multirow{2}{*}{$1 \times 1 \times 1(10$ штук $)$} & $\begin{array}{c}9.2 \times 1.2 \times 1.2,2.2 \times 1.2 \times 1.2 \\
2.2 \times 1.2 \times 1.2,1.2 \times 1.2 \times 1.2\end{array}$ & $\approx 1$ час \\
\hline \multirow{2}{*}{$1 \times 1 \times 1(10$ штук $)$} & $\begin{array}{c}4.2 \times 1.2 \times 1.2,2.2 \times 1.2 \times 1.2, \\
2.2 \times 1.2 \times 1.2,1.2 \times 1.2 \times 1.2\end{array}$ & $\approx 2.5$ часа \\
\hline
\end{tabular}

Разработанная технология позволяет найти точное решение за приемлемое с точки зрения практики время не более чем для 10-15 грузов (например, время решения задачи размещения 15 одинаковых грузов размера $1 \times 1 \times 1$ в 1 реальном контейнере размера $14.2 \times 1.2 \times 1.2$ составило около 11 часов). Вычислить оптимальное распределение большего количества грузов за разумное время удалось только в специальных случаях - в частности, для решения задачи размещения 30 грузов размера $1 \times 1 \times 1$ в 29 реальных контейнерах размера $1.2 \times 1.2 \times 1.2$ потребовалось около 20 минут, а для решения задачи размещения 100 грузов размера $1 \times 1 \times 1$ в 1 реальном контейнере размера $10 \times 10 \times 10$ - около 5 минут.

\section{5. Обсуждение предложенного подхода. Практическая значимость}

К явным достоинствам разработанной технологии решения задачи ОЗВТ стоит отнести возможность применения стандартных методов решения за- 
дач СЦЛП для поиска ее экстремума. Построенная модель схожа с предложенными в [11-13], но требует вдвое меньше бинарных переменных для формализации условий отсутствия пересечения грузов. Если в указанных работах число переменных-индикаторов того, что один из грузов находится левее/правее, выше/ниже, сзади/спереди другого груза, равно $3 n(n-1)$, то в предложенной модели общее количество переменных $a_{i_{1}, i_{2}}, b_{i_{1}, i_{2}}, c_{i_{1}, i_{2}}\left(i_{1}=\right.$ $\left.=1, \ldots, n-1, i_{2}=i_{1}+1, \ldots, n\right)$ равно $3 n(n-1) / 2$. Кроме того, использование единого координатного пространства для всех контейнеров позволяет избежать необходимости формировать блоки ограничений, записываемых для каждой пары грузов и каждого контейнера (например, см. неравенства (7) в [11]).

$\mathrm{C}$ точки зрения демонстрации и популяризации результатов проведенных работ описанную технологию выгодно отличает реализация в форме открытого сервиса, доступного в сети Интернет.

Дополнительным достоинством является возможность интеграции в предложенную технологию методов и средств многокритериального анализа альтернатив, применяемых для выбора предпочтительных ЛА.

К явным недостаткам разработанной технологии, учитывая NP-трудность задачи ОЗВТ, необходимо отнести значительное время и мощные вычислительные ресурсы, которые могут потребоваться для решения (при большом количестве контейнеров и грузов время, необходимое для поиска оптимума, будет неприемлемым с практической точки зрения). Тем не менее стоит заметить, что предложенная технология может быть применена для верификации и калибровки разрабатываемых приближенных эвристических подходов к решению задачи ОЗВТ на тестовых примерах небольшой размерности. Кроме того, на основе предложенной аналитической модели могут быть разработаны методы аппроксимации точного решения задачи O3ВТ. Например, нижние оценки могут быть рассчитаны с помощью эвристических алгоритмов поиска допустимых точек построенной задачи СЦЛП [19], а верхние оценки могут быть рассчитаны, в частности, путем решения линейных релаксационных задач, полученных удалением ряда ограничений из задачи ОЗВТ.

В построенной формализации нет ограничений, связанных с тем, что незакрепленные дополнительными техническими средствами грузы должны надежно опираться на другой груз либо на пол контейнера. В результате расчета может оказаться, что предметы частично или полностью висят в воздухе. Перспективным подходом к решению данной проблемы представляется добавление в целевую функцию слагаемого, представляющего собой взвешенную сумму координат грузов по оси $z$, взятую со знаком «минус». 
Направления дальнейших исследований включают в себя модификацию формализации задачи с целью учета пределов допустимого давления на грузы, необходимости обеспечения устойчивости предметов, требований группировки заданных наборов грузов, более сложных форм контейнеров, а также других ограничений, возникающих на практике. Кроме того, возрастающая сложность рассматриваемых задач (вследствие добавления новых ограничений и увеличения количества контейнеров и грузов) потребует разработки дополнительных эвристических подходов, которые бы позволили строить на основе сформированной модели достаточно эффективные варианты загрузки (не обязательно являющиеся глобально оптимальными). В перспективе разработанная технология может стать основой для составной части комплекса, функционал которого будет нацелен на решение более сложных задач, например, подразумевающих формирование расписаний авиаперевозок при ограничениях на количество, характеристики и доступность ЛА, ограничений на летные условия, ограничений на сроки доставки и т.д. [20].

\section{6. Заключение}

В ходе выполнения исследования рассмотрена задача оптимизации загрузки упорядоченной совокупности ЛА при ряде допущений относительно параметров грузов и принципов их размещения в отсеках. Данная задача математически формализована как задача СЦЛП, что позволяет использовать для ее решения точные алгоритмы. Разработана и реализована (в форме открытого Интернет-сервиса) технология решения указанной задачи, основанная на использовании оптимизатора SCIP. Адекватность работы предложенной технологии проверена на совокупности тестовых примеров малой размерности.

Практическое применение представленной технологии в перспективе позволит снизить общие финансовые затраты на воздушную транспортировку грузов, сократит время погрузочно-разгрузочных работ, а также поспособствует обеспечению сохранности перевозимых товаров.

Определены основные направления дальнейшего развития разработанного программного комплекса, нацеленные на расширение спектра задач, которые можно будет решать с его помощью.

\section{СПИСОК ЛИТЕРАТУРЫ}

1. E.E. Bischoff, M.S.W. Ratcliff. Issues in the development of approaches to container loading // Omega, The International Journal of Management Science, 1995, v.23. №4, p.377-390.

2. A. Bortfeldt, G. Wäscher. Constraints in container loading - A state-of-the-art review // European Journal of Operational Research, 2013, v.229, №1, p.1-20. 
3. J. Egeblad, C. Garavelli, S. Lisi, D. Pisinger. Heuristics for container loading of furniture // European Journal of Operational Research, 2010, v.200, №3, p.881-892.

4. C. Paquay, M. Schyns, S. Limbourg. A mixed integer programming formulation for the three-dimensional bin packing problem deriving from an air cargo application // International Transactions in Operational Research, 2016, v.23, №1-2, p.187-213.

5. M.R. Garey, D.S. Johnson. Computers and intractability: a guide to the theory of NPcompleteness. - W. H. Freeman and Company, San Francisco, 1979.

6. В.В. Псиола. О приближенном решении трехмерной задачи об упаковке на основе эвристик // Интеллектуальные системы, 2007, т.11, вып.1-4, с.83-100;

V.V. Psiola. O priblizhennom reshenii trekhmernoi zadachi ob upakovke na osnove evristik // Intellektualnye sistemy, 2007, t.11, vyp.1-4, s.83-100.

7. M. Eley. A bottleneck assignment approach to the multiple container loading problem // OR Spectrum, 2003, v.25, №1, p.45-60.

8. A. Lim, H. Ma, J. Xu, X. Zhang. An iterated construction approach with dynamic prioritization for solving the container loading problems // Expert Systems with Applications, 2012, v.39, №4, p.4292-4305.

9. Z. Jin, T. Ito, K. Ohno. The three-dimensional bin packing problem and its practical algorithm // JSME International Journal Series C, 2003, v.46, №1, p.60-66.

10. C.H. Che, W. Huang, A. Lim, W. Zhu. The multiple container loading cost minimization problem // European Journal of Operational Research, 2011, v.214, №3, p.501-511.

11. C.S. Chen, S.M. Lee, Q.S. Shen. An analytical model for the container loading problem // European Journal of Operational Research, 1995, v.80, №1, p.68-76.

12. M. Padberg. Packing small boxes into a big box // Mathematical Methods of Operations Research, 2000, v.52, №1, p.1-21.

13. H.T. Hong Ha, N. Nananukul. Air cargo loading management system for logistics forwarders // Proceedings of 2016 International Conference on Urban Planning, Transport and Construction Engineering (ICUPTCE'16), Pattaya, Jan. 2-3, 2016, p.51-58.

14. L. Junqueira, R. Morabito, D.S. Yamashita. Three-dimensional container loading models with cargo stability and load bearing constraints // Computers \& Operations Research, 2012, v.39, №1, p.74-85.

15. В.П. Осипов, В.А. Судаков. Комбинированный метод поддержки принятия многокритериальных решений. - М.: ИПМ им. М.В. Келдыша РАН, 2015, препринт №30, 21 с.;

V.P. Osipov, V.A. Sudakov. Kombinirovannyi metod podderzhki priniatiia mnogokriterialnyh reshenii. - M.: IPM im. M.V. Keldysha RAN, 2015, preprint №30, 21 s.

16. B. Korte, J. Vygen. Combinatorial optimization: theory and algorithms. - Springer-Verlag Berlin Heidelberg, 2006.

17. T.C. Hu. Integer programming and network flows. - Addison-Wesley Publishing Company, Reading, Massachusetts, 1970.

18. J.T. Linderoth, T.K. Ralphs. Noncommercial software for mixed-integer linear programming // Integer Programming: Theory and Practice, CRC Press Operations Research Series, 2005, p.253-303.

19. T. Achterberg. SCIP: solving constraint integer programs // Mathematical Programming Computation, 2009, v.1, №1, p.1-41.

20. H. Pollaris, K. Braekers, A. Caris, G.K. Janssens, S. Limbourg. Vehicle routing problems with loading constraints: state-of-the-art and future directions // OR Spectrum, 2015, v.37, iss.2, p.297-330. 